NASA/TM-1999-209444

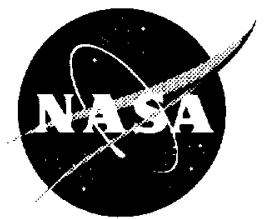

\title{
Porous Media and Mixture Models for Hygrothermal Behavior of Phenolic Composites
}

Roy M. Sullivan

Glenn Research Center, Cleveland, Ohio

Eric H. Stokes

Southern Research Institute, Birmingham, Alabama

Prepared for the

International Mechanical Engineering Congress and Exposition sponsored by the American Society of Mechanical Engineers

Nashville, Tennessee, November 14-19, 1999

National Aeronautics and Space Administration

Glenn Research Center 


\section{Available from}

NASA Center for Aerospace Information 7121 Standard Drive

Hanover, MD 21076

Price Code: A03
National Technical Information Service 5285 Port Royal Road Springfield, VA 22100 Price Code: A03 


\title{
POROUS MEDIA AND MIXTURE MODELS FOR HYGROTHERMAL BEHAVIOR OF PHENOLIC COMPOSITES
}

\author{
Roy M. Sullivan \\ National Aeronautics and Space Administration \\ Glenn Research Center \\ Cleveland, $\mathrm{OH} 44135$ \\ Eric H. Stokes \\ Southern Research Institute \\ Birmingham, AL 35211
}

\section{ABSTRACT}

Theoretical models are proposed to describe the interaction of water with phenolic polymer. The theoretical models involve the study of the flow of a viscous fluid through a porous media and the thermodynamic theory of mixtures. From the theory a set of mathematical relations are developed to simulate the effect of water on the thermostructural response of phenolic composites. The expressions are applied to simulate the measured effect of water in a series of experiments conducted on carbon phenolic composites.

\section{NOMENCLATURE}

A Specific Helmholtz Free Energy

$A_{i} \quad$ Partial Helmholtz. Free Energy of $\mathrm{i}^{\text {th }}$ Constituent

E Polymer Network Stiffness

$M_{p}^{R U} \quad$ Molecular Weight of the Polymer Repeating Unit

$M_{w} \quad$ Molecular Weight of Water

$N_{p} \quad$ Number of Moles of Polymer Molecules per Unit Volume

$N_{p}^{R U} \quad$ Number of Moles of Polymer Repeating Unit per Unit Volume

$N_{w} \quad$ Number of Moles of Water Molecules per Unit Volume

$P \quad$ Solid Mixture Pressure

$P_{i} \quad$ Partial Pressure of $\mathrm{i}^{\text {th }}$ Constituent

$P_{t(t)} \quad$ Total Pressure on the Material

$T \quad$ Temperature

$\tilde{\mathbf{v}}_{w} \quad$ Water Vapor Velocity Vector

$g \quad$ Specific Gibbs Free Energy

$\begin{array}{ll}g_{i} & \text { Partial Gibbs Free Energy of } i^{\text {th }} \text { Constituent } \\ k & \text { Permeability } \\ p & \text { Pore Vapor Pressure } \\ p_{w} & \text { Vapor Pressure of Water } \\ s & \text { Specific Entropy } \\ s_{i} & \text { Partial Entropy of } i^{\text {th }} \text { Constituent } \\ v & \text { Specific Volume } \\ v_{i} & \text { Partial Volume of } i^{\text {th }} \text { Constituent } \\ \alpha & \text { Thermal Expansion Coefficient for Polymer Network } \\ \varepsilon_{n} & \text { Material Strain Normal to the Fabric Plane } \\ \varepsilon_{n}^{\text {w }} & \text { Material Strain Normal to the Fabric Plane due to } \\ \phi & \text { Water } \\ \mu & \text { Porosity } \\ \rho & \text { Vapor Viscosity } \\ \rho_{i} & \text { Density } \\ v_{i} & \text { Martial Density of } i^{\text {it }} \text { Constituent }\end{array}$

Subscripts

n material direction normal to the fabric plane

p polymer

w water

Superscripts

p Pore Volume

s Solid Mixture 


\section{INTRODUCTION}

Phenolic composites are used as thermal insulation in a variety of high temperature applications. Phenolic composites are quite often fabricated as a laminate structure using a carbon or ceramic woven fabric reinforcement. Although these materials char and ablate at ultra high temperatures, their structural integrity must be maintained at moderately high temperatures in order to fulfill their protective function.

The thermostructural response of the phenolic composite material is significantly influenced by the presence of water in the material. The effect of water on the thermal expansion hehavior and the thermal stresses generated in carbon fabric reinforced phenolic composites has been measured by Stokes (1987) and Hubbert (1989). This effect is most pronounced in the direction normal to the fabric plane, the direction which is most significantly influenced by the physical events occurring in the polymer.

In this paper, we propose theoretical models, which describe the interaction of water with the phenolic composite material. These theoretical models involve the study of porous media and the flow of a viscous fluid through a porous media. We also employ mixtures theory and thermodynamic principles of condensed mixtures to develop mathematical relations for simulating the effect of water on the thermostructural response. The mathematical relations are applied to simulate the measured effect of water on the thermal expansion and thermal stress response of carbon fabric reinforced phenolic composites. In the end. the mathematical relations prove to be quite accurate in simulating the effect of water as measured by Stokes (1987) and Hubbert (1989).

\section{MATHEMATICAL MODELS}

\section{Basic Assumptions: A Review of the Paradigm}

In order to develop the mathematical theory to describe the effect of water on the thermostructural behavior, let us consider the scenario which is depicted by Fig. 1. The water molecules (represented by circles), given their polar nature. occupy charged sites on the polymer chains resulting from local induced or permanent dipole formations on the phenolic network. It's also possible that clusters of water molecules are associated with local concentrations of charged sites on the thermoset resin. As the polymer network is heated past the glass transition temperature. it will undergo significant elongation. When the water molecules have absorbed enough energy to overcome the bond energy, they will be liberated from the polymer chains and essentially evaporate into the free volumes of the polymer (Fig. 1). The effusion of the water molecules from the material occurs through the flow of water molecules through the free volume of the polymer. The water molecules that remain attached to the polymer network have a significant impact on the thermal expansion behavior of the polymer network and on the thermal stresses that are generated in the material.

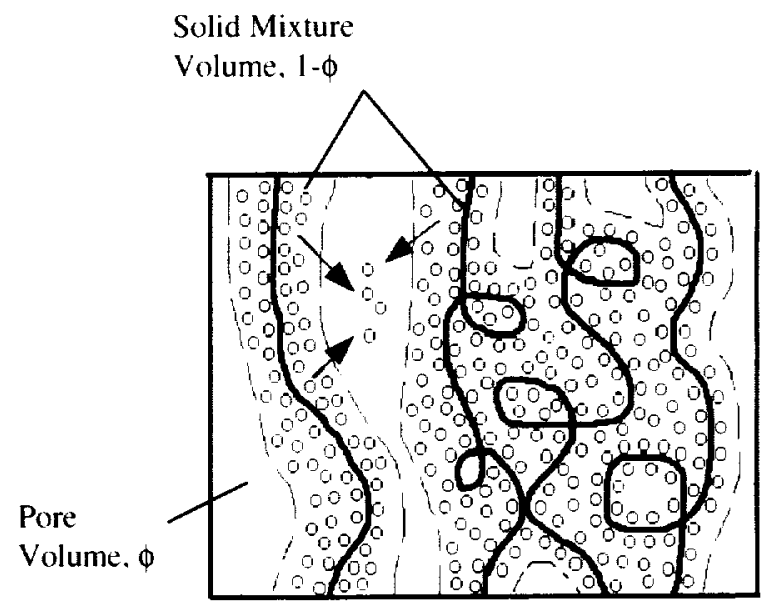

Fig.1. Sketch of the Polymer Containing Water

We propose that these physical processes are sufficiently represented by a porous media model where water is evaporated from a solid skeleton into a pore network and that, once evaporated, the water flows in the form of water vapor through the pore network. The polymer free volume is represented by the pore volume with volume fraction $\phi$. The polymer and attached water molecules are represented by the solid skeleton and occupy the volume $1-\phi$. We assume that the evaporation of water molecules from their sites on the solid skeleton is thermodynamically equivalent to the evaporation of water molecules from a condensed mixture, a mixture where only the water constituent is volatile. The force responsible for the water vapor flow though the pore network is the pore pressure gradient.

This thermodynamic system may be represented by the sketch shown in Fig. 2. Each cell in the sketch represents an open thermodynamic system consisting of a condensed mixture of polymer and water in equilibrium with its vapor. Each mixture is itself a homogeneous mixture, although the concentration may vary from cell to cell. Water may enter or exit each cell through the pore network in the form of water vapor. Within each local thermodynamic system (cell), chemical equilibrium between the water molecules in the mixture and the water molecules in the vapor state is always maintained. Water may either evaporate from or condense into the mixture depending upon the local concentration of water, the temperature and the vapor pressure. 


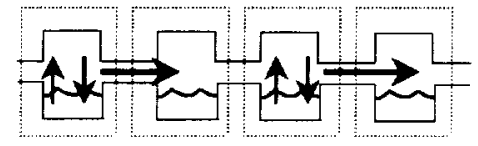

Fig. 2. Sketch of the Equivalent Thermodynamic System

As water will exist at both the solid sites and in the pore volumes in the form of water vapor, the volume average expression for the density of water in the composite material is

$$
\rho_{u}=(1-\phi) \rho_{u}^{s}+\phi \rho_{u}^{p}
$$

where $\rho_{w}^{p}$ is the density of the water in the pores. $\rho_{w}^{s}$ is the density of the water in the solid mixture and $\phi$ is the volumetric porosity.

\section{Thermodynamics of Mixtures: Derivation of the} Mixture Pressure Expression

The thermodynamic relations for incremental changes in the specific Helmholtz free energy and specific Gibbs free energy for a homogeneous body are given by

$$
\begin{aligned}
& d A=-s d T-P d V \\
& d g=-s d T+v d P
\end{aligned}
$$

where A, g, s, v, T and $\mathrm{P}$ are the specific Helmholtz free energy, the specific Gibbs free energy, the specific entropy, the specific volume, the temperature and the pressure, respectively.

For a homogeneous mixture of two constituents. the usual form of the mixtures relations for the extensive variables are

$$
\begin{aligned}
& \rho A=\rho_{1} A_{1}+\rho_{2} A_{2} \\
& \rho g=\rho_{1} g_{1}+\rho_{2} g_{2} \\
& \rho s=\rho_{1} s_{1}+\rho_{2} s_{2}
\end{aligned}
$$

where $\rho$ is the density of the mixture and where $\rho_{1}$ and $\rho_{2}$ are the partial densities of constituents $I$ and 2 , respectively. In equations (4) through (6), $A_{1}, g_{1}$ and $s_{1}$ are the partial Helmholtz free energy, the partial Gibbs free energy and the partial entropy of constituent 1 , respectively. The quantities $A_{2}$. $g_{2}$ and $s_{2}$ are the partial Helmholtz free energy, the partial Gibbs free energy and the partial entropy of constituent 2. respectively. The differential form of (5) can be written as

$$
\rho d g=\rho_{1} d g_{1}+\rho_{2} d g_{2}
$$

provided the density and partial densities do not vary considerably over the differential increment.

The same relations as given in equations (2) and (3) can be used 10 relate the partial thermodynamic quantities for each constituent in the mixture. That is the partial Helmholtz free energies and the partial Gibbs free energies for constituents 1 and 2 are given by

$$
\begin{aligned}
& d A_{1}=-s_{1} d T-P_{1} d v_{1} \\
& d A_{2}=-s_{2} d T-P_{2} d v_{2} \\
& d g_{1}=-s_{1} d T+v_{1} d P_{1} \\
& d g_{2}=-s_{2} d T+v_{2} d P_{2}
\end{aligned}
$$

where $v_{1}$ and $v_{2}$ are partial volumes and $P_{1}$ and $P_{2}$ are the partial pressures.

Rewriting (3) and multiplying through by the density of the mixture $\rho$, we get

$$
\rho v d P=\rho d g+\rho s d T
$$

Substituting (6) and (7) into (12), we obtain

$$
\rho v d P=\rho_{1} d g_{1}+\rho_{2} d g_{2}+\rho_{1} s_{1} d T+\rho_{2} s_{2} d T .
$$

Finally. upon substituting (10) and (11) into (13), we obtain

$$
\rho v d P=\rho_{1} v_{1} d P_{1}+\rho_{2} v_{2} d P_{2} .
$$

Since the density of the mixture is the inverse of the specific volume, $\rho v=1$. Equation (14) therefore becomes

$$
d P=\rho_{1} v_{1} d P_{1}+\rho_{2} v_{2} d P_{2} .
$$

Equation (15) expresses the total pressure acting on the mixture in terms of the partial pressures of the two constituents. 
Note that the partial densities $\rho_{i}$ are the mass of the ith constituent per unit total volume, whereas the partial volumes $v_{i}$ are the volumes occupied by each unit mass of the ith constituent as measured within the occupied volumes. As such, the products $\rho_{1} v_{1}$ and $\rho_{2} v_{2}$ represent volume fractions of constituent 1 and 2, respectively and (15) may be rewritten as

$$
d P=v_{1} d P_{1}+v_{2} d P_{2}
$$

where $v_{i}$ are the volume fractions.

In the application of the mixtures theory to the mixture of phenolic polymer and water, the subscripts 1 and 2 in equations (15) and (16) are replaced with $w$ and $p$ to obtain

$$
d P=\rho_{w}^{s} v_{w} d P_{w}+\rho_{p} v_{p} d P_{p}
$$

and

$$
d P=v_{n} d P_{n}+v_{p} d P_{P}
$$

In (17), the superscript $s$ has been added to $\rho_{w}$ to denote the density of water in the condensed (solid) mixture.

\section{Application of Mixtures Theory to the RTG Experiments}

The Restrained Thermal Growth (RTG) test was designed to measure the thermal stress generated in the material while subjected to high temperatures and simultaneously restrained from expansion in one of the principal material directions. In the tests conducted by Stokes (1987) and Hubbert (1989), the stress (or pressure) in the direction normal to the fabric plane which is required to maintain zero strain in that direction was recorded as a function of temperature.

1. We note that (16) has precisely the form of the volume average stress expression used in porous media theory to express the total stress of a porous body $\tilde{\boldsymbol{\sigma}}$ in terms of the solid skeleton stress $\tilde{\sigma}^{\prime}$ and the pore volume stress $\tilde{\sigma}^{\prime}$. namely $\tilde{\sigma}=(1-\phi) \tilde{\sigma}^{\prime}+\phi \tilde{\sigma}^{p}$ where $\phi$ is the volumetric porosity. It should be noted, however, that equation (16) is not of the same form as Dalton:s law of partial pressures which defines the tolal pressure of gaseous mixtures as the sum of the partial pressures, $P=P_{1}+P_{2}+\ldots$. Equation (16) would have the same form as Dalton's law if each gas constituent simultaneously occupies the same volume, namely the total volume. since in that case $v_{1}=v_{2}=v_{3}=\ldots=1$
In order to apply the mixtures theory to simulate the effect of water in the RTG experiment, it is necessary to make certain assumptions regarding the thermodynamic state of the water in the material. After all, equations (17) and (18) do not specify the variation of the partial pressure with temperature, but merely relate the partial pressures to the total pressure. Knowing how the thermodynamic state of the water in the polymer/water mixture varies with temperature will necessarily specify the variation of its partial pressure with temperature.

To this end, we first assume that as the mixture is heated through a continuous series of equilibrium states, the partial Gibbs free energy of the water does not vary. $d g_{w}=0$. Under this condition, equation (10) can be rewritten as

$$
s_{n} d T=v_{w} d P_{w}
$$

after replacing the subscript 1 with $w$ to represent the water constituent. Substituting (19), equation (17) may be written

$$
d P=\rho_{w}^{s} s_{u} d T+\rho_{p} v^{\prime}{ }_{p} d P_{p}
$$

The total pressure is obtained by integration of $(20)$ as

$$
P=P^{\prime \prime}+\int \rho_{w}^{s} s_{u} d T+\int \rho_{p} v_{p} d P_{p}
$$

where $P^{\prime \prime}$ is the total pressure at some reference temperature.

The last term in equation (21) represents the response of a RTG specimen devoid of water since it represents the pressure response when $\rho_{w}^{s}=0$. The second term on the right hand side in (21) represents the additional stress due to the presence of water. This term. of course. accounts for the variation in moisture content through the value of $\rho_{u}^{\prime}$.

We also make the assumption that $s_{\mathfrak{w}}$ in (21) is the entropy of condensed water, which follows from our paradigm represented by Fig. 2 that the mixture in each cell is condensed. The variation of $s_{w}$, with temperature follows the variation of the entropy of condensed water with temperature.

\footnotetext{
2. Equation (19) implies that the increment in the complimentary heat absorbed by the water is equal to the increment in the complimentary work performed by the water.
} 
In Fig. 3, the restraining stress predicted by equation (21) is plotted as a function of temperature along with measured RTG results from Hubbert (1989). The measured results are from tests conducted on FM5055 carbon phenolic specimens heated at a constant rate of $5.55^{\circ} \mathrm{C} / \mathrm{sec}\left(10^{\circ} \mathrm{F} / \mathrm{sec}\right)$. The measured results are shown for specimens with $0 \%, 4 \%$ and $8 \%$ initial moisture and are plotted as dashed lines. The responses predicted by equation (21) for $4 \%$ and $8 \%$ moisture are plotted as solid lines. This comparison has been shown previously in Sullivan (1996).

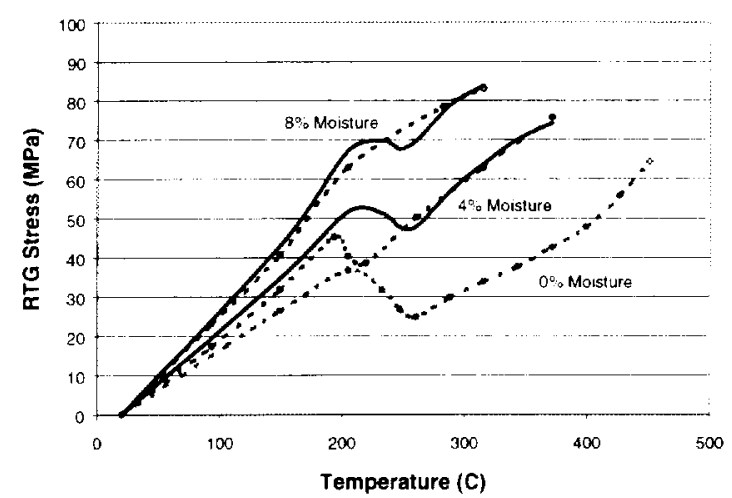

Fig. 3. Comparison of Measured and Predicted RTG Results

Our assumption that the thermodynamic state of water molecules in the polymer network may be approximated by the thermodynamic state of condensed water results in close agreement between the predicted and measured RTG results. This assumption seems justified in light of the fact that, in the RTG experiment, the mechanical constraint and the presence of the polymer molecules restrict the movement of the water molecules, limit their available volume and maintain a low entropy state which is approximately equal to that of condensed water. Furthermore, we may argue that, under the constraints imposed in the RTG experiment, there is no significant free molar volume available for the evaporation of water molecules.

\section{Definition of Volume Fractions}

The thermodynamic theory of polymer solutions utilizes a liquid lattice model to establish relations for the entropy of mixing in polymer solutions (Flory, 1953). In the lattice model, every site in the liquid lattice is assumed to be occupied by either a solvent molecule or a segment of the polymer chain. Using the lattice model, the volume fractions for the water and polymer in the mixture may be written as

$$
v_{u}=\frac{N_{w}}{N_{n}+x N_{p}}
$$

and

$$
v_{p}=\frac{x N_{p}}{N_{x}+x N_{p}},
$$

respectively, where $N_{\mathrm{u}}$ is the number of moles of water per unit volume and $N_{p}$ is the number of moles of polymer molecules per unit volume. The quantity $x$ is defined as the ratio of molar volumes of polymer and water. Its presence in the volume fraction relations is necessary in order to account for the large differences in molar volumes between the two constituents.

If we assign $x$ to be the average number of repeating units in the polymer chains., then the number of moles of the repeating units per unit volume $N_{p}^{R U}$ is given by $N_{p}^{R U}=x_{p}$. The water volume fraction is therefore given by

$$
v_{w}=\frac{N_{w}}{N_{w}+N_{p}^{R U}} .
$$

The volume fraction now has the form of a mole fraction. since we have accounted for the relative molecular size of the two constituents

The number of moles of water and polymer repeating unit per unit volume in the mixture are related to the partial densities by $N_{1}=\rho_{w}^{s} / M_{w}$, and $N_{p}^{R U}=\rho_{p} / M_{p}^{R U}$, respectively, where $M_{w}$ is the molecular weight of water and $M_{p}^{R U}$ is the molecular weight of the repeating unit. The water volume fraction may therefore be written in terms of the partial densities of water and polymer as

$$
v_{u^{\prime}}=\frac{\rho_{w}^{s}}{\rho_{w^{\prime}}^{s}+\bar{M} \rho_{p}}
$$

where $\bar{M}=M_{w^{\prime}} / M_{p}^{R U}$. Alternatively, we may write

$$
\rho_{w}^{s}=\frac{v_{w}}{1-v_{w}} \bar{M} \rho_{p} .
$$

3. This definition is not the same as the previous definition of $x$. However, the two definitions are consistent if we assume that each site in the liquid lattice is occupied by either a water molecule or a polymer repeating unit. i.e. that the repeating unit is the fundamental mixing unit of the polymer. 


\section{Porous Media Model for Evaporation and Flow}

For an ideal gas llowing through a porous solid body, the local mass conservation equation is written as

$$
\phi \frac{\partial \rho_{w}^{p}}{\partial t}+\tilde{\nabla} \cdot\left(\rho_{w}^{p} \tilde{\mathbf{v}}_{w}\right)=-(1-\phi) \frac{\partial \rho_{w}^{s}}{\partial t}
$$

where $\tilde{\mathbf{v}}_{w}$ is the average velocity vector for the water and $\partial() / \partial t$ denotes the time derivative. In equation (26), the first term represents the time rate of change of pore water density per unit bulk volume. The second term represents the rate of change in pore water density per unit bulk volume due to flow through the pores. The last term represents the rate at which water is supplied to the pores by evaporation from the solid.

In order to determine the rate at which water evaporates from the solid, we consider a thermodynamic system consisting of a condensed mixture containing water in chemical equilibrium with its vapor. As this mixture is heated through a continuous series of equilibrium states. chemical equilibrium between the mixture and its vapor leads to the relation

$$
d v_{w}=v_{w}\left\{\frac{d p}{p}-\frac{d p_{w}}{p_{w}}\right\}
$$

where $p_{n}$ is the vapor pressure of pure water and $p$ represents the pressure of the vapor. Equation (27) results from differentiation of Raoult s law. $p=v_{w} p_{w}{ }^{4}$.

Using (27), the time derivative of the water volume fraction may be written as

$$
\frac{\partial v_{w}}{\partial t}=v_{w}\left\{\frac{1}{p} \frac{\partial p}{\partial t}-\frac{1}{p_{w}} \frac{\partial p_{w}}{\partial T} \frac{\partial T}{\partial t}\right\}
$$

Using the chain rule of differentiation, we have

$$
\frac{\partial \rho_{w}^{s}}{\partial t}=\frac{\partial \rho_{w}^{s}}{\partial v_{w}} \frac{\partial v_{w}}{\partial t}
$$

4. The classic expression of Raoult's law uses a mole fraction rather than a volume fraction. The use of the volume fraction instead of the mole fraction comes from the fact that. in polymer solutions. the volume fraction is a more accurate representation of the activity (Flory. 1953).
Differentiating (25) with respect to $v_{w}$ and using (28) and (24), equation (29) becomes

$$
\frac{\partial \rho_{u}^{s}}{\partial t}=\frac{\rho_{w}^{s}\left(\rho_{w}^{s}+\bar{M} \rho_{p}\right)}{\bar{M} \rho_{p}}\left\{\frac{1}{p} \frac{\partial p}{\partial t}-\frac{1}{p_{w}} \frac{\partial p_{w}}{\partial T} \frac{\partial T}{\partial t}\right\} .
$$

Substituting ( 30 ), equation ( 26 ) becomes

$$
\begin{aligned}
\phi \frac{\partial \rho_{w}^{p}}{\partial t}+ & \tilde{\nabla} \cdot\left(\rho_{n}^{p} \tilde{\mathbf{v}}_{w}\right)= \\
& \quad(1-\phi) \frac{\rho_{w^{s}}^{s}\left(\rho_{w^{\prime}}^{s}+\bar{M} \rho_{p}\right)}{\bar{M} \rho_{p}}\left\{\frac{1}{p} \frac{\partial p}{\partial t}-\frac{1}{p_{w}} \frac{\partial p_{w^{\prime}}}{\partial T} \frac{\partial T}{\partial t}\right\}
\end{aligned}
$$

Substituting the ideal gas relation for the pore volume density, $\rho_{w}^{p \prime}=p / R T$, into equation (31) leads finally to

$$
\begin{gathered}
\left\{\phi \rho_{w}^{p}+\frac{(1-\phi) \rho_{w}^{s}\left(\rho_{w}^{s}+\bar{M} \rho_{p}\right)}{\bar{M} \rho_{p}}\right\} \\
p \\
\frac{\partial p}{\partial t}+\tilde{\nabla} \cdot\left(\rho_{w}^{p} \tilde{\mathbf{v}}_{w}\right) \\
=\left\{\frac{\phi \rho_{w}^{p}}{T}+\frac{(1-\phi) \rho_{w}^{s}\left(\rho_{w}^{s}+\bar{M} \rho_{p}\right)}{\bar{M} \rho_{p}} \frac{1}{p_{w}} \frac{\partial p_{w}}{\partial T}\right\} \frac{\partial T}{\partial t} .
\end{gathered}
$$

The water vapor velocity vector $\tilde{\mathbf{v}}_{w}$ is calculated using Darcy's law, which is given by $\tilde{\mathbf{v}}_{\mathfrak{w}}=-(k / \mu) \tilde{\nabla} \cdot p$ where $k$ is the permeability and $\mu$ is the viscosity of the vapor.

\section{Numerical Simulation of Water Effusion in the Thermogravimetric Experiments}

Thermogravimetric tests involved heating a sample of material in an atmosphere of inert gas and simultaneously recording the sample's weight as a function of temperature. The data generated is the weight fraction remaining versus temperature. Tests were conducted on cylindrical samples which were $0.635 \mathrm{~cm}(0.25$ inches $)$ in diameter and $1.27 \mathrm{~cm}$

\footnotetext{
5. The use of the volume fraction in Raoult's law results in a slightly more complicated relation for $\partial \rho_{\mathrm{w}}^{\prime} / \partial t$ than that given in Sullivan and Stokes (1997) where the water mass concentration was used to relate the pore vapor pressure to the vapor pressure of pure water.
} 
( 0.50 inches) long. The samples were heated radiantly with the electrical resistance heater, the exact heating rate being controlled manually or with an electronic temperature controller. Each run consisted of a $20^{\circ} \mathrm{C} /$ minute ramp $10100^{\circ} \mathrm{C}$, followed by a 10 -minute hold at $100^{\circ} \mathrm{C}$, and finally a linear ramp rate of $3^{\circ} \mathrm{C} /$ minute. A detailed description of the test procedure and thermogravimetric facility are given in Stokes (1993, 1994a \& 1994b).

The weight loss data as a function of thermocouple temperature was fitted with a cubic spline. Derivative weight loss curves were produced for each run by taking the derivative of the spline curve with respect to temperature. A derivative weight loss curve consists of a series of peaks, each peak representing a weight loss event.

Thermogravimetric tests were conducted on specimens with $0 \%$ moisture and $4 \%$ moisture. The derivative weight loss curves for all the $0 \%$ moisture specimens were averaged to obtain an average $0 \%$ derivative weight loss curve. The derivative weight loss curves for all the $4 \%$ moisture specimens were averaged to obtain an average $4 \%$ derivative weight loss curve. The net derivative weight loss curve was obtained by subtracting the average $0 \%$ derivative weight loss curve from the average $4 \%$ derivative weight loss curve. As such, the net derivative weight loss curve consists of one peak. which represents the weight loss due to the effusion of moisture from the $4 \%$ moisture specimen.

Equation (32) is used to model the effusion of water from carbon phenolic specimens during the thermogravimetric experiments. We assume that the flow of water in the cylindrical specimens is purely radial. The one-dimensional. axisymmetric form of equation (32) is cast into a matrix equation using the finite element method and solved numerically at discrete time increments. The details of the finite element approach for solving (32) and the numerical solution scheme are described in Sullivan and Stokes (1997).

The solution to equation (32) yields the pore vapor pressures at the finite element nodes at each time step. The average pore vapor pressure in each finite element is obtained by averaging the element nodal values. The water volume fraction is updated for each finite element using (27). The partial density of water in the solid mixture $\rho_{\mathfrak{w}}^{s}$ is updated for each finite element using equation (25). The density of water vapor in the pores $\rho_{n}^{\prime \prime}$ is calculated from the pore vapor pressure and the temperature using the ideal gas law. The time step is then incremented and the new temperature is calculated. The vapor pressure of pure water $p_{w}$ and the temperature derivative $d p_{n} / d T$ are updated for the new temperature using a tabular input. The values for these quantities as a function of temperature were obtained from Keenan, et al., (1969). The solution to (32) is performed again and the process is repeated until the end of the simulation.

The results of the thermogravimetric simulation are shown in Figs. 4 through 6 . These numerical results were obtained by using the numerical values for porosity, permeability and molecular weight of the repeating unit listed in Table 1.

Table 1. List of Properties Used for Thermogravimetric Simulation

\begin{tabular}{|c|c|c|}
\hline Property & Value & Reference \\
\hline$\phi(\%)$ & 11.5 & Stokes $(1992 \mathrm{a})$ \\
\hline$k(\mathrm{sq} . \mathrm{cm})$. & $2.69 \times 10^{-1 \hbar}$ & Stokes $(1992 \mathrm{~b})$ \\
\hline$M_{p}^{R I}(\mathrm{~g} / \mathrm{mole})$ & 107 & Theoretical \\
\hline
\end{tabular}

The pore vapor pressure as a function of spatial position in the specimen at various temperatures is plotted in Fig. 4. Since the specimens are cylindrical, the pore vapor pressure is plotted versus the radial position using a non-dimensional radius ratio. A radius ratio value of zero represents the centerline of the cylindrical specimen and a radius ratio value of unity represents the outer radius of the specimen. At temperatures below $150^{\circ} \mathrm{C}$. the pore vapor pressure is uniform throughout the specimen. Ahove $200^{\circ} \mathrm{C}$, the vapor pressure no longer remains uniform due to water vapor flow through the pore network. From room temperature to $300^{\circ} \mathrm{C}$, the pore vapor pressure increases with increasing temperature. Above $300^{\circ} \mathrm{C}$, the vapor pressure decreases with increasing temperature due to the depletion of moisture in the specimens. Less water in the solid mixture results in lower pore vapor pressures.

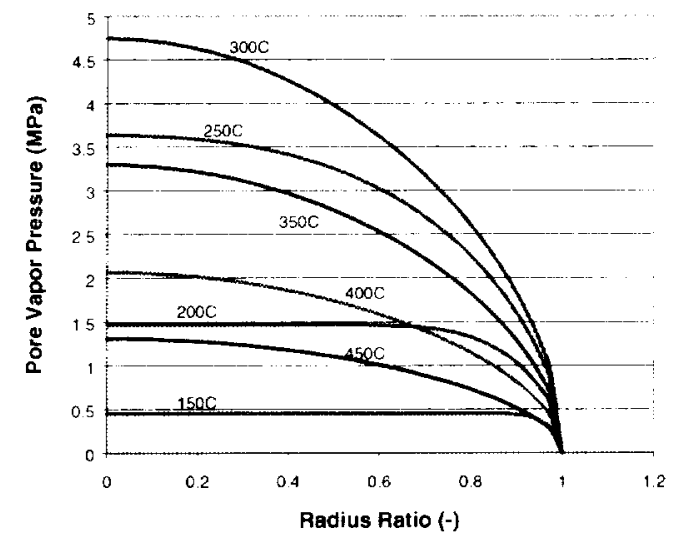

Fig. 4. Predicted Pore Vapor Pressure Versus Radial Position at Various Temperatures for the Thermogravimetric Experiment 
The water volume fraction and the water density in the solid skeleton were volume averaged at each time step. The volume average density of water in the solid skeleton and the volume average water volume fraction are plotted as a function of temperature in Fig. 5. These results indicate that a significant amount of evaporation from the solid mixture begins at approximately $200^{\circ} \mathrm{C}$ and that all of the water is evaporated above $400^{\circ} \mathrm{C}$.

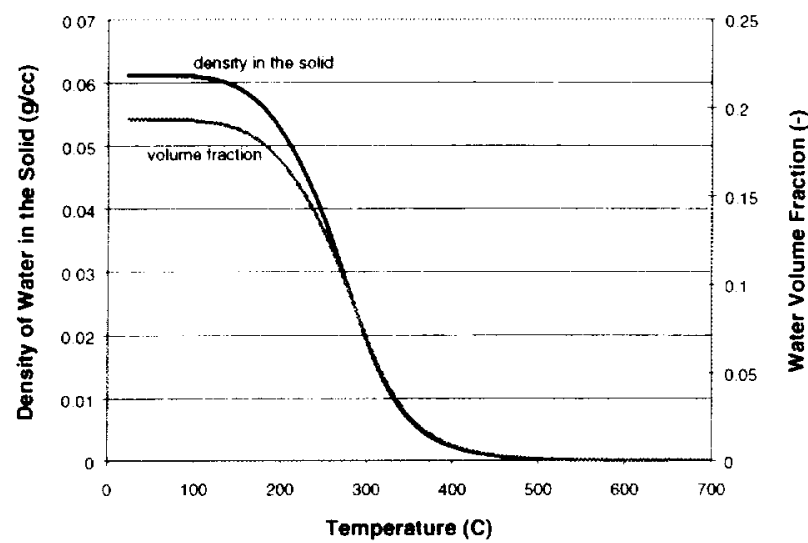

Fig. 5. Predicted Average Density of Water in the Solid Mixture and Average Water Volume Fraction Versus Temperature in Thermogravimetric Experiment

The mathematical theory for evaporation and flow and the numerical solution scheme are verified in Fig. 6, where the derivative weight loss obtained from the numerical solution is compared to the measured, net derivative weight loss reported in Sullivan and Stokes (1997). The numerical solution results are plotted as a solid line and the measured results are plotted as hollow circles. The derivative weight loss is obtained from the numerical solution by taking the temperature derivative of equation (1) using the volume average values for $\rho_{w}^{s}$ and $\rho_{w}^{p}$. Notice the close correlation between the measured net derivative weight loss and that predicted by the numerical simulation.

\section{Mechanics of Free Expansion}

The thermal expansion of FM5055 carbon phenolic was measured by Stokes (1987). The thermal expansion in the direction normal to the fabric plane is shown in Fig. 7 for specimens containing $0 \%, 4 \%$ and $8 \%$ moisture and heated at $5.55^{\circ} \mathrm{C} / \mathrm{sec}\left(10^{\circ} \mathrm{F} / \mathrm{sec}\right)$. Cylindrical specimens $0.635 \mathrm{~cm}(0.25$ inch) in diameter and $2.54 \mathrm{~cm}$ ( 1.0 inch) long were used for the thermal expansion measurements. Notice the significant effect of water content on the thermal expansion behavior.

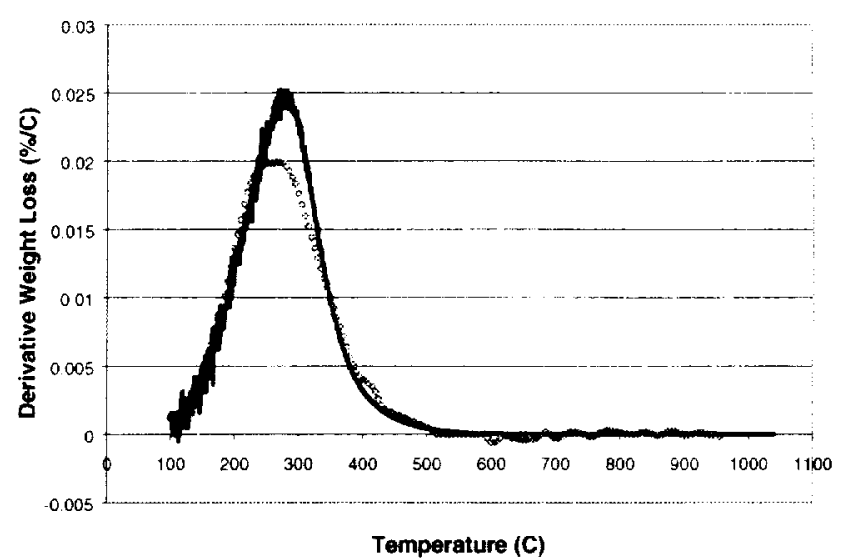

Fig. 6. Comparison Between Measured and Predicted Derivative Weight Loss

The thermal expansion response normal to the fabric plane may be separated into two parts: the inherent thermal expansion behavior, due solely to the thermal excitation of the polymer network, and that which is due to the presence of water in the polymer network. This is expressed mathematically as

$$
\varepsilon_{n}=\int \alpha_{n} d T+\varepsilon_{n}^{n}
$$

where the first term on the right hand side represents the inherent thermal expansion behavior of the polymer network and the second term represents the expansion strain due to the presence of water in the network. The thermal expansion response of the $0 \%$ moisture specimen shown in Fig. 7 is the inherent thermal expansion behavior of the polymer network. The strain due to water $\varepsilon_{n}^{\prime \prime}$ is dependent upon the amount of water in the material and may be obtained from the thermal expansion measurements as the difference between the $0 \%$ specimen response and the response of the $4 \%$ and $8 \%$ specimens.

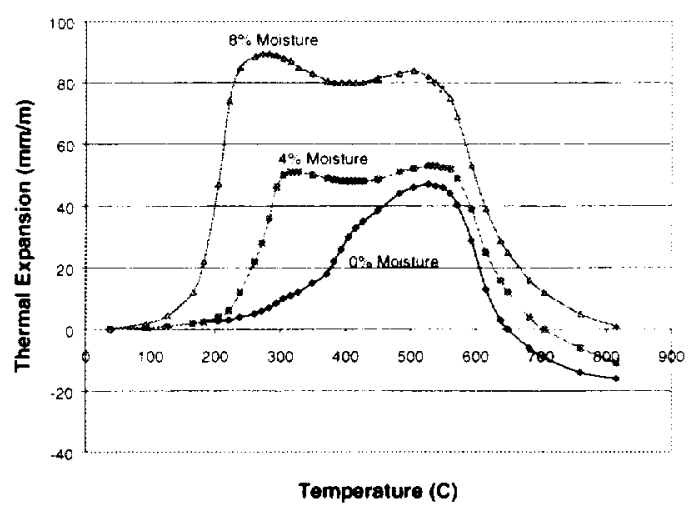

Fig. 7. Measured Thermal Expansion Versus Temperature for Various Moisture Contents 
In order to establish an expression for $\varepsilon_{n}^{w}$. we note that the differential increment in the total pressure of the material $d P_{t o t}$ may be given by the volume average expression as

$$
d P_{t o r}=(1-\phi) d P+\phi d p
$$

where again $P$ is the pressure of the solid mixture and $p$ is the pore vapor pressure. We also note that, during the free expansion tests, the increment in the total pressure normal to the fabric plane is zero. Setting equation (34) to zero and neglecting the pore vapor pressure term leads to $d P=0^{\circ}$. Using (18), we may obtain

$$
d P_{p}=-\frac{v_{n}}{1-v_{n}} d P_{w}
$$

since $v_{w}+v_{p}=1$. Thus, although the total stress increment is zero, the partial pressure in the polymer network is not. This polymer network partial pressure is the source of $\varepsilon_{n}^{n \text {. }}$.

Assuming that the polymer network is linear elastic and restricting our attention to one-dimensional expansion, the polymer network strain normal to the fabric plane $\varepsilon_{n}^{n}$ is given by $\varepsilon_{n}^{\prime \prime}=P_{p} / E_{n}$ where $E_{n}$ is the polymer network stiffness normal to the fabric plane ${ }^{8}$. The strain can thus be written in terms of the water partial pressure and the volume fraction as

$$
\varepsilon_{n}^{u}=\frac{1}{E_{n}} \int \frac{v_{u}}{\left(1-v_{w}\right)} d P_{w} .
$$

Once again. we must assume the thermodynamic state of water in the mixture in order to estimate the variation of its partial pressure with temperature $P_{w}=P_{w}(T)$. Again, we

6. The relative insignificance of the pore vapor pressure is illustrated by comparing the results shown in Figs. 3 and 4. The pore vapor pressure values shown in Fig. 4 are less than 5 MPa. whereas the calculated partial pressures of water in the mixture. as evidenced by Fig. 3. are significantly higher.

7. The negative sign results since the water partial pressure is compressive and the polymer partial pressure is tensile.

8. The normal stiffness $E_{n}$ is also dependent upon the amount of water in the polymer network, since water will effect the degree of cross-linking in the polymer network and will have an effect on the glass transition temperature in glassy polymers. The latter effect is evident in Fig. 7.

9. In (36). we have dropped the negative sign since it has already been acknowledged that $P_{11}$ is compressive. assume that as the water/polymer mixture is heated, the partial Gibbs free energy of water in the mixture remains constant. This leads again to the relation given in (19). Substituting (19) into (36) leads to

$$
\varepsilon_{n}^{n}=\frac{1}{E_{n}} \int \frac{v_{w}}{\left(1-v_{w}\right)} \frac{s_{w}}{v_{w}} d T
$$

where $s_{w}$ and $v_{w}$ are assumed to be the entropy and partial volume of condensed water. respectively.

\section{Numerical Simulation of the Effect of Water in the Thermal Expansion Measurements}

We will now use the previously discussed theories to model the effect of water on the thermal expansion response of carbon phenolic. We use the evaporation and flow model to calculate the amount of water in the thermal expansion specimens as a function of time and spatial location. Again, we assume that the effusion of water from the specimen occurs through a pore network, that the pore vapor pressure gradients drive the effusion of water from the specimen and that the water vapor flow in the cylindrical specimens is purely radial.

The pore vapor pressures, the density of water in the solid mixture and the water volume fractions are calculated at each time step in the same manner as described in the previous section. The normal tensile strain $\varepsilon_{n}^{\prime \prime}$ is calculated by equation (37) at each time step using a volume average value for the water volume fraction. The numerical values for $s_{w}$ and $v_{w}$ as a function of temperature was obtained from Keenan. et al. (1969). The tensile stiffness $E_{n}$ was obtained from measurements made by Stokes (1987. 1990a. 1990) $)$. The variation of $E_{n}$ with temperature for both $4 \%$ and $8 \%$ initial moisture contents is plotted in Fig. 8 . The numerical values listed in Table 1 for the porosity. permeability and molecular weight of the repeating unit are once again used for the simulation.

In Fig. 9. the numerical results for $\varepsilon_{n}^{\prime \prime}$ obtained hy (37) are plotted as a function of temperature and compared to the measured responses. The measured response for $\varepsilon_{11}^{\prime \prime}$ for the $4 \%$ and $8 \%$ moisture specimens was obtained by subtracting the thermal expansion response for the $0 \%$ moisture specimens from the thermal expansion response for the $4 \%$ and $8 \%$ moisture, respectively. The numerical results are plotted as solid lines and the measured responses are plotted as dashed lines with hollow circles. 


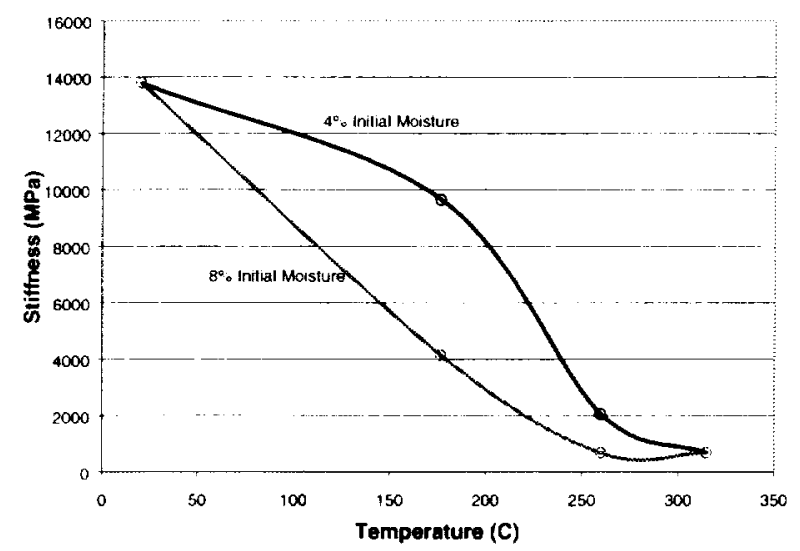

Fig. 8. Tensile Stiffness Versus Temperature for $4 \%$ and $8 \%$ Moisture

At temperatures below $250^{\circ} \mathrm{C}$. the strain response obtained by the numerical solution and equation (37) compares well with the measured response of the $8 \%$ moisture specimens. The predicted results for the $4 \%$ specimen overestimates the measured response at all temperatures. For both moisture contents. the peak in the measured strain response occurs at lower temperatures and lower strain values than that predicted by the theory and numerical solution. The reason for this discrepancy is that the permeability was held constant in the numerical solution. whereas, in reality, the permeability is a function of strain state. The effect of strain on permeability has been measured by Stokes $(1992 \mathrm{c})$. As the material strains. the permeability will increase. This will cause the moisture flow rates to increase. The end result is the exhaustion of moisture from the specimens at temperatures lower than that predicted by the present solution.

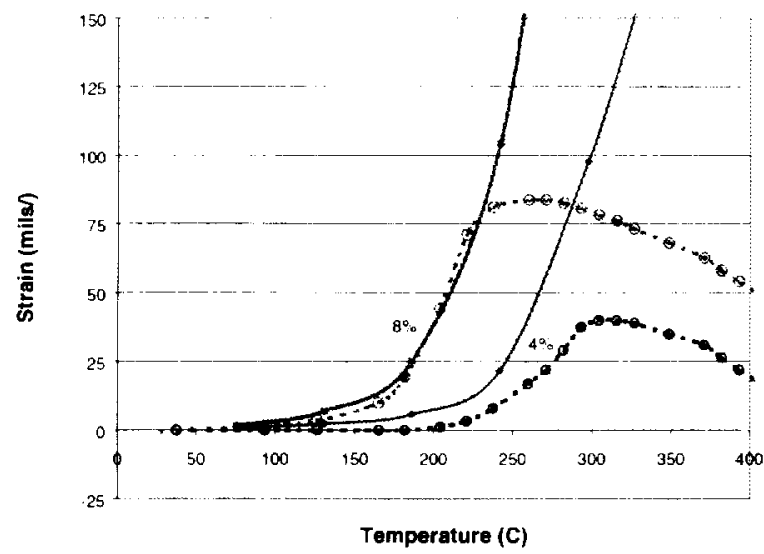

Fig. 9. Comparison Between Measured and Predicted Normal Strains Versus Temperature

\section{DISCUSSION}

Theoretical models have been proposed which describe the interaction of water with phenolic polymer. These models are proposed in order to derive a set of equations which simulate the hygrothermal behavior of reinforced phenolic composites.

The evaporation and flow equation was successful in simulating the effusion behavior of water from FM5055 carbon phenolic specimens during thermogravimetric experiments. This equation was derived from porous media principles as well as from the thermodynamic principles of condensed mixtures. It was developed based on the assumptions that the liberation of water molecules from the polymer is thermodynamically equivalent to the evaporation of water from a condensed mixture and that the bound water on the polymer network is in chemical equilibrium with the free water in the free molar volumes. Furthermore, it was assumed that the water transport occurs through the free molar volumes.

In treating the polymer network and the water molecules which are attached to the polymer network as a condensed, binary mixture. two equations were derived from the thermodynamics of mixtures principles. One equation simulates the thermal stresses generated by the water. The other simulates the stress-induced thermal strains due to water. The accuracy of these two equations was examined by applying them to simulate the effect of water in the RTG and thermal expansion experiments. In both cases. the response predicted by the theoretical models was in close agreement with the measured response.

\section{REFERENCES}

Hubbert. T. E., 1989, "Mechanical Properties of FM5055 Baseline Carbon Phenolic." SRI-MME-89-1149-6071-23.

Flory, P. J.. 1953. Principles of Polymer Chemistry, Cornell University Press, New York.

Keenan, J. H., Keyes, F. G., Hill, P. G. and Moore, J. G.. 1969. Steam Tables: Thermodynamic Properties of Water Including Vapor, Liquid and Solid Phases, John Wiley and Sons, New York.

Stokes. E. H., 1987, "The Effect of Moisture on the Mechanical and Thermal Response of FM 5055 Carbon Phenolic Composite." SRI-EAS-87-1244-6245-6.

Stokes, E. H., 1988, "The Physical, Mechanical and Thermal Properties of the FM 5055404 Aft Inlet Ring. Thermal Tests, Gas Permeahility and Thermal Expansion. Part 1." SRI-EAS88-201-6032-1, March. 252 p. 
Stokes, E. H., 1990a, "Across Ply Mechanical and Thermal Characteristics of FM 5055 (Avtex Pre-Shutdown) as a Function of the Material's Moisture Content" SRI-MME-901109-6526-5.

Stokes, E. H., 1990b. "Moisture Effects on Across Ply Mechanical and Thermal Characteristics of FM 5055 Carbon Phenolic Utilizing Rapid Isothermal Heating," In: Proceedings JANNAF Propulsion Meeting. Anaheim, CA. CPIA Pub. 550. pp. 505-512.

Stokes, E. H., 1992a, "Anomalous Swelling Behavior of FM 5055 Carbon Phenolic Composite," In: Proceedings 33rd AIAA/ASME/ASCE/AHS/ASC Structures, Structural Dinamic's and Materials Conference, pp. 2932-2938, AIAA Paper 922428. April. see also: AIAA Joumal, Vol. 31. No. 3. March. 1993, pp. 584-589.

Stokes, E. H., 1992b, "Permeability of Carbonized Rayon Based Polymer Composites," In: Proceedings ASME Simposium on Computational Mechanics of Porous Solid Materials and Their Thermal Decomposition. Scottsdale, AZ. AMD-Vol. 136, April. pp. 145-156.
Stokes, E. H., 1992c, "Gas Permeability of RSRM Carbon Phenolic Composites as a Function of Across Ply Tensile Stress, Strain, and Temperature," In: Proceedings JANNAF-RNTS Meeting. Sunnyvale, CA, edited by M. J. Paul, CPIA Pub. 592. December. pp. 457-466.

Stokes, E. H.. 1993, "Kinetics of Pyrolysis Mass Loss From Phenolic Resin Based Composites." In: Proceedings JANNAFRNTS Meeting. Huntsville. AL, edited by M. J. Paul, CPIA Pub. 605, October, pp. 235-246.

Stokes, E. H., 1994a, "Mass Loss from Phenolic Resin Based Composites Subjected to High Heating Rates." In: Proceedings JANNAF-RNTS Meeting, Seattle, WA, edited by M. J. Paul, CPIA Pub. 618, November 15-17, pp. 367-379.

Stokes, E.H.. 1994b. "Kinetics of Pyrolysis Mass Loss from MX 4926 Standard Density NARC Based Carbon Phenolic Composite," SRI-MME-94-043-6672.18A.

Sullivan, R. M.. 1996, "The Effect of Water on Thermal Stresses in Polymer Composites," ASME Joumal of Applied Mechanics, Vol. 63, pp. 173-179.

Sullivan, Roy M. and Stokes, Eric H., 1997, "A Model for the Effusion of Water in Carbon Phenolic Composites," Mechanics of Materials. Vol. 26, pp. 197-207. 
Public reporting burden for this collection of intormation is estimated to average 1 hour per response, including the time for reviewing instructions, searching existing data sources, gathering and mantaining the data needed. and completing and reviewing the collection of information. Send comments regarding this burden estimate or any other aspect of this collection of intormation, including suggestions for reducing this burden. to Washington Headquarters Services. Directorate for Information Operations and Reports. 1215 Jefferson

Davis Highway. Sutte 1204. Artington, VA 22202-4302, and to the Office of Management and Budget, Paperwork Reduction Project (0704-0188). Washington. DC 20503.

\begin{tabular}{|l|l|l}
\hline 1. AGENCY USE ONLY (Leave blank) & $\begin{array}{c}\text { 2. REPORT DATE } \\
\text { October } 1999\end{array}$ & $\begin{array}{r}\text { 3. REPORT TYPE AND DATES COVERED } \\
\text { Technical Memorandum }\end{array}$
\end{tabular}

4. TITLE AND SUBTITLE

Porous Media and Mixture Models for Hygrothermal Behavior of Phenolic Composites

6. AUTHOR(S)

Roy M. Sullivan and Eric H. Stokes

\section{PERFORMING ORGANIZATION NAME(S) AND ADDRESS(ES)}

National Aeronautics and Space Administration

John H. Glenn Research Center at Lewis Field

Cleveland, Ohio 441.35-3191

WU-242-23-5.3-00

8. PERFORMING ORGANIZATION REPORT NUMBER

$E-11930$

9. SPONSORING/MONITORING AGENCY NAME(S) AND ADDRESS(ES)

National Aeronautics and Space Administration

Washington. DC 20546-0001

10. SPONSORING/MONITORING AGENCY REPORT NUMBER

NASA TM-1999-209444

\section{SUPPLEMENTARY NOTES}

Prepared for the International Mechanical Engineering Congress and Exposition sponsored by the American Society of Mechanical Engineers. Nashville. Tennessee. November 14-19. 1999. Roy M. Sullivan, NASA Glenn Research Center. and Eric H. Stokes. Southern Research Institute. Birmingham, Alabama 35211. Responsible person,

Roy M. Sullivan. organization code 5920, (216) 433-3249.

12a. DISTRIBUTION/AVALABILITY STATEMENT

\section{2b. DISTRIBUTION CODE}

Unclassitied - Unlimited

Subject Categories: $15,20,24,25$ and 77

Distribution: Nonstandard

This publication is available from the NASA Center for AcroSpace Information. (301) 621-0390.

13. ABSTRACT (Maximum 200 words)

Theoretical models are proposed to describe the interaction of water with phenolic polymer. The theoretical models involve the study of the flow of a viscous fluid through a porous media and the thermodynamic theory of mixtures. From the theory a set of mathematical relations are developed to simulate the effect of water on the thermostructural response of phenolic composites. The expressions are applied to simulate the measured effect of water in a series of experiments conducted on carbon phenolic composites.

14. SUBJECT TERMS

Carbon phenolic composites: Thermodynamics of mixtures; Porous media;

Thermogravimetric analysis; Moisture effusion 17. SECURITY CLASSIFICATION
OF REPORT

Unclassified

\section{SECURITY CLASSIFICATION OF THIS PAGE}

Unclassified
19. SECURITY CLASSIFICATION OF ABSTRACT

Unclassified

\begin{tabular}{l} 
15. NUMBER OF PAGES \\
17 \\
\hline $\begin{array}{c}\text { 16. PAICE CODE } \\
\text { A03 }\end{array}$
\end{tabular}

20. LIMITATION OF ABSTRACT

Standard Form 298 (Rev. 2-89) Prescribed by ANSI Std. Z39-18 298-102 